\title{
Physiotherapy contributions to weaning and extubation of patients from mechanical ventilation
}

\author{
D Morar, MSc Physiotherapy; H van Aswegen, $\mathrm{PhD}$ \\ Department of Physiotherapy, School of Therapeutic Sciences, Faculty of Health Sciences, University of the Witwatersrand, Johannesburg, South Africa
}

Corresponding author: $H$ van Aswegen (helena.vanaswegen@wits.ac.za)

\begin{abstract}
Background. Liberation of patients from mechanical ventilation (MV) is an important goal of patient care, to avoid the complications and risks associated with prolonged MV.

Objective. To determine the extent of South African physiotherapists' involvement in weaning and extubation of patients from MV and whether current practice is evidence based.

Method. A survey questionnaire was developed, and content validated and made available electronically and in hard copy. Physiotherapists working in adult intensive care units in public and private sector hospitals in South Africa (SA) were identified and invited to participate.

Results. Response rate was $43 \%(n=184)$. The majority of respondents $(n=135,73 \%)$ 'never' or 'seldom' got involved in decision-making to wean patients from MV; a minority $(n=8,4 \%)$ were 'routinely' involved in decision-making. Some respondents $(n=54,29 \%)$ performed extubation 'often' or 'routinely'. The majority used exercises $(n=149,81 \%)$, early mobilisation out of bed $(n=142,77 \%)$ and deep breathing exercises (DBEs) $(n=142,77 \%)$ 'routinely' to aid in respiratory muscle training. The majority of respondents 'never' adjusted ventilator settings other than fraction of inspired oxygen. No association was found between type of physiotherapy degree respondents held and their involvement in weaning $(p=0.24)$.

Conclusion. SA physiotherapists' contributions towards weaning of patients from MV through prescription of exercise therapy, early outof-bed mobilisation and DBEs is evidence based. Involvement in adjustment of MV settings, decision-making regarding patient weaning, development of weaning protocols for their units and extubation is limited.
\end{abstract}

S Afr J Crit Care 2016;32(1):6-10. DOI:10.7196/SAJCC.2016.v32i1.254

Management of patients with critical illness often includes intubation and mechanical ventilation (MV) to assist patients through periods of exhaustion and poor gas exchange resulting from respiratory failure. Liberation of patients from MV is an important goal of patient care to avoid the complications and risks associated with prolonged MV, such as diaphragm dysfunction, dyspnoea and hypercapnia, physical deconditioning and muscle weakness, exercise intolerance, increased risk for morbidity and mortality, prolonged intensive care unit (ICU) length of stay and reduced health-related quality of life. ${ }^{[1-3]}$ Weaning involves either abrupt or gradual withdrawal of MV support. ${ }^{[4]}$ Reducing MV support too quickly, however, may result in fatigue or cardiovascular instability, either of which may delay the weaning process.

Physiotherapists' involvement in weaning of patients from MV includes provision of muscle-strengthening exercises, early mobilisation, administration of checklists to assess patient readiness for weaning, and identification of and treatment for patients receiving non-invasive ventilation (NIV) ${ }^{[3,5-9]}$ In the South African (SA) critical care setting, physiotherapists not only provide care to patients in the form of pulmonary therapy, exercise and mobilisation interventions but are also actively involved in extubation of patients. ${ }^{[10]}$ A pilot study by Van Aswegen and Potterton ${ }^{[10]}$ on the scope of physiotherapy practice in ICU showed, however, that SA physiotherapists had little involvement in weaning of patients from MV. More recently, Plani et al., ${ }^{[1]]}$ in a single-centred, non-randomised experimental trial, investigated the effect of a nurse- and physiotherapist-driven weaning and extubation protocol on MV duration and ICU length of stay of patients with traumatic injuries in an 'open'-type ICU in SA. They reported a clinically significant reduction in MV duration and proposed that the role of SA physiotherapists and ICU nurses in weaning and extubation of patients in 'open'-type ICUs should be expanded. ${ }^{[1]}$ In an attempt to begin addressing this issue, the authors set out to: (i) establish the extent of involvement of physiotherapists nationwide in weaning patients from MV and (ii) describe current physiotherapy practice related to weaning and extubation.

\section{Methods}

A descriptive, quantitative, survey-based study was performed. Ethics clearance was received from the University of the Witwatersrand Human Research (Medical) Ethics Committee prior to commencement (clearance no.:M110901). A surveyquestionnaire was developedbased on theavailable literature regarding the involvement of physiotherapists in weaning and extubation of patients on MV. Content validation of the questionnaire was performed in January 2012, whereby a panel consisting of two experienced cardiopulmonary physiotherapy lecturers and two senior physiotherapists with vast ICU working experience critically analysed each question in the questionnaire. Each panellist was emailed the questionnaire and study aims and objectives 2 weeks prior to the meeting with the researchers, to familiarise themselves with the documents. At the meeting, suggestions and points of improvement were noted. Once these changes were made, the questionnaire was emailed back to the panel members for review. After consensus was reached on the final version of the questionnaire, it was loaded electronically onto SurveyMonkey for data collection. The final version of the questionnaire consisted of the following sections: demographics and ICU categorisation; weaning protocol; adjustment of mechanical ventilator settings; weaning and extubation; weaning interventions and physiotherapy modalities; physiotherapy autonomy; and education (qualification of respondent, existing opportunities for ongoing professional development and interest in further training).

Physiotherapists who worked in adult ICUs in the public or private healthcare sectors were invited to participate in the survey. 
Physiotherapists who did not work in ICU, physiotherapy assistants, undergraduate physiotherapy students and community service physiotherapists were excluded.

The process of obtaining contact details for public and private sector hospitals lasted from March to July 2012. Firstly, the Department of Health was contacted to determine the name and location of all public sector hospitals in SA that had ICU facilities. The head of each public hospital physiotherapy department was contacted telephonically and, with permission via email, given information about the survey. This included a description of the purpose of the survey as well as inclusion and exclusion criteria. Heads of departments were asked to discuss the survey with their staff and whether email or postal surveys were preferred as the method of questionnaire delivery. Secondly, the Hospital Association of SA was contacted to source the main private hospitals under the Netcare, Mediclinic and Life hospital brands. Each private hospital ICU was contacted to acquire the names and contact details of private physiotherapy practices that served the ICU. After permission was obtained from private practice owners to share their information with the researchers, each practice was contacted telephonically, with permission via email, to share information about the survey. Practice owners were asked to share the information with their staff. Lastly, the national chairperson of the SA Society of Physiotherapy Cardiopulmonary Physiotherapy Rehabilitation Special Interest Group (CPRG) was contacted regarding the purpose of and criteria for the survey, and asked to share this information with CPRG members.

Once heads of departments and private practice owners had identified staff members who were interested in participating and who fit the criteria for the survey, a list of email and postal addresses of participants was compiled by the researchers. The electronic link to the survey was emailed to participants. Hardcopy questionnaires with self-addressed return envelopes were posted to those who requested such. All hardcopy questionnaires were coded. Once the CPRG chairperson gave consent to approach CPRG members about the survey, the researchers sent the relevant documents and electronic link to the survey to the CPRG secretary, who disseminated the information to their membership.

Participants were given 2 months to complete the survey. A courtesy email was sent 2 - 4 weeks after the initial email to thank participants who had completed the online survey and remind those who had not to do so by the due date. A courtesy call was made, at similar time intervals, to departments and participants who requested hardcopy questionnaires to determine whether they received the questionnaires and to remind participants to complete and return the questionnaires by the due date. All completed questionnaires received via post or electronically were included and analysed.

\section{Statistical analysis}

Descriptive statistics were used for analysis using Stata statistics and data analysis software version 11 (StataCorp, USA). Continuous data were summarised as means and standard deviations (SDs). Categorical data were summarised as frequencies and percentages. Fischer's exact test was used to determine the involvement of physiotherapists in weaning in relation to number of years qualified and type of physiotherapy degree held. Logistic regression was used to determine if the number of years qualified had an influence on respondents' involvement in weaning. A $p$-value $<0.05$ was deemed statistically significant.

\section{Results}

A total of 425 questionnaires were distributed among physiotherapists in SA who met the inclusion criteria. The response rate for postal questionnaires was $55 \%(n=109)$ and for the online survey was $33 \%(n=75)$, resulting in a combined response rate of $43 \%(n=184)$. Table 1 summarises demographic information about the respondents and their work environments. The majority of respondents were senior physiotherapists or managers $(n=119,65 \%)$ and most respondents worked in the public healthcare sector $(n=120,66 \%)$. Most respondents had worked in an ICU for between 1 and 5 years $(n=113,62 \%)$, with only $11 \%(n=20)$ reporting $\geq 16$ years' ICU work experience. Most $(n=155,91 \%)$ held a Bachelor of Science degree in physiotherapy. Most respondents reported that they worked in an 'open' ICU setting, and the majority worked in mixed medical, surgical and trauma ICUs. An open-type ICU was defined as an ICU that was nonspecific physician led. Thirty-eight per cent of respondents $(n=69)$ reported that their ICU had a guideline or protocol for weaning in place; however, only $16 \%(n=11)$ of respondents were involved in the development of these guidelines or protocols.

The majority of respondents 'never' adjusted ventilator settings related to ventilation mode, respiratory rate, tidal volume, inspiratory
Table 1. Demographics of respondents and their work environment at the time of the survey

\begin{tabular}{|c|c|}
\hline Variable & $n(\%)$ \\
\hline \multicolumn{2}{|l|}{ Position held $(N=183)$} \\
\hline $\begin{array}{l}\text { Junior physiotherapist } \\
\text { (<3 years' work experience) }\end{array}$ & $64(35)$ \\
\hline $\begin{array}{l}\text { Senior physiotherapist } \\
\text { ( } \geq 3 \text { years' work experience) }\end{array}$ & $10(5)$ \\
\hline Head of department & $79(43)$ \\
\hline Practice owner & $30(16)$ \\
\hline \multicolumn{2}{|l|}{ Years qualified $(N=184)$} \\
\hline $1-5$ & $95(52)$ \\
\hline $6-10$ & $39(21)$ \\
\hline $11-15$ & $24(13)$ \\
\hline$\geq 16$ & $26(14)$ \\
\hline \multicolumn{2}{|l|}{$\begin{array}{l}\text { Years of ICU work experience } \\
(N=183)\end{array}$} \\
\hline $1-5$ & $113(62)$ \\
\hline $6-10$ & $40(22)$ \\
\hline $11-15$ & $9(5)$ \\
\hline$\geq 16$ or more & $21(11)$ \\
\hline \multicolumn{2}{|l|}{$\begin{array}{l}\text { Type of qualification held by } \\
\text { respondents }(N=171)\end{array}$} \\
\hline Diploma in Physiotherapy & $7(4)$ \\
\hline $\begin{array}{l}\text { Bachelor of Science in } \\
\text { Physiotherapy }\end{array}$ & $155(91)$ \\
\hline $\begin{array}{l}\text { Master of Science in } \\
\text { Physiotherapy }\end{array}$ & $9(5)$ \\
\hline Doctor of Philosophy & $0(0)$ \\
\hline \multicolumn{2}{|l|}{ Healthcare sector $(N=183)$} \\
\hline Public & $120(66)$ \\
\hline Private & $63(34)$ \\
\hline \multicolumn{2}{|l|}{ Classification of ICU* } \\
\hline Open & 165 \\
\hline Closed & 85 \\
\hline \multicolumn{2}{|l|}{ Type of $\mathrm{ICU}^{\dagger}$} \\
\hline Surgical & 23 \\
\hline Cardiac/cardiothoracic & 37 \\
\hline Trauma & 15 \\
\hline Medical & 19 \\
\hline Neurology & 23 \\
\hline Burns & 10 \\
\hline Mixed medical and surgical & 23 \\
\hline $\begin{array}{l}\text { Mixed medical, surgical and } \\
\text { trauma }\end{array}$ & 123 \\
\hline${ }^{*}$ More than one ICU per hospital & \\
\hline${ }^{\top}$ Respondents worked in more than or & \\
\hline
\end{tabular}

pressure and positive end-expiratory pressure (PEEP) (Fig. 1). It appears that respondents were more involved in adjusting the fraction of inspired oxygen $\left(\mathrm{FiO}_{2}\right)$ setting on the 
ventilator. This is reflected by $12 \%(n=22)$ reporting that they 'routinely' adjusted $\mathrm{FiO}_{2}$; $11 \%(n=20)$ reported they 'often' made this adjustment and $14 \%(n=26)$ reported that they 'frequently' adjusted $\mathrm{FiO}_{2}$.

Seventy-three per cent $(n=135)$ of respondents indicated that they 'never' or 'seldom' got involved in decision-making to start weaning a patient from $\mathrm{MV}$, in contrast to a minority $(n=8 ; 4 \%)$ who were 'routinely' involved in such decision-making. The majority of respondents ( $n=113,61 \%)$ were 'seldom' or 'never' involved in providing advice to the interprofessional team in ICU on extubation of patients. Some respondents $(n=54,29 \%)$ performed extubation 'often' or 'routinely'. The majority of respondents $(n=119,65 \%)$ indicated that they 'never' implemented a spontaneous breathing trial to facilitate weaning of a patient from MV. Similarly, many respondents ( $n=101,55 \%)$ 'never' implemented NIV in patient care after extubation.

Fig. 2 shows which physiotherapy modalities were used by respondents to facilitate respiratory muscle strengthening in patients who are mechanically ventilated. The majority of respondents $(n=149,81 \%)$ used exercises 'routinely' in the form of active-assisted and active exercises to aid in respiratory muscle training. Many respondents used early mobilisation out of bed $(n=142,77 \%)$ and DBE ( $n=142,77 \%)$ 'routinely'. Interventions not used 'often' were DBE with biofeedback from the ventilator, DBE with adjustment of pressure support (PS) levels and DBE with manual hyperinflation (MHI). The most common modality 'never' used by respondents to strengthen respiratory muscles was DBE with adjustment of PS $(n=125,68 \%)$. This is in keeping with a majority of respondents ( $n=158,86 \%)$ who reported to 'never' adjust the PS setting on the ventilator (Fig. 1).

Physiotherapy autonomy was defined as the ability of respondents to make decisions on the weaning of a patient from MV in the unit where they worked and implementation of the decision without direct supervision from a medical colleague. Respondents were asked to rate their autonomy using a Likerttype scale. A mean (SD) rate of 1.9 (1.6) was calculated, where ' 1 ' correlated to no autonomy and ' 10 ' to complete autonomy. When asked how often respondents' contributions influenced decisions regarding MV in their unit, a mean rate of 2.9 (2.2) was recorded. On this scale, ' 1 ' correlated to never and ' 10 ' to always.

There was no association between the number of years respondents were qualified

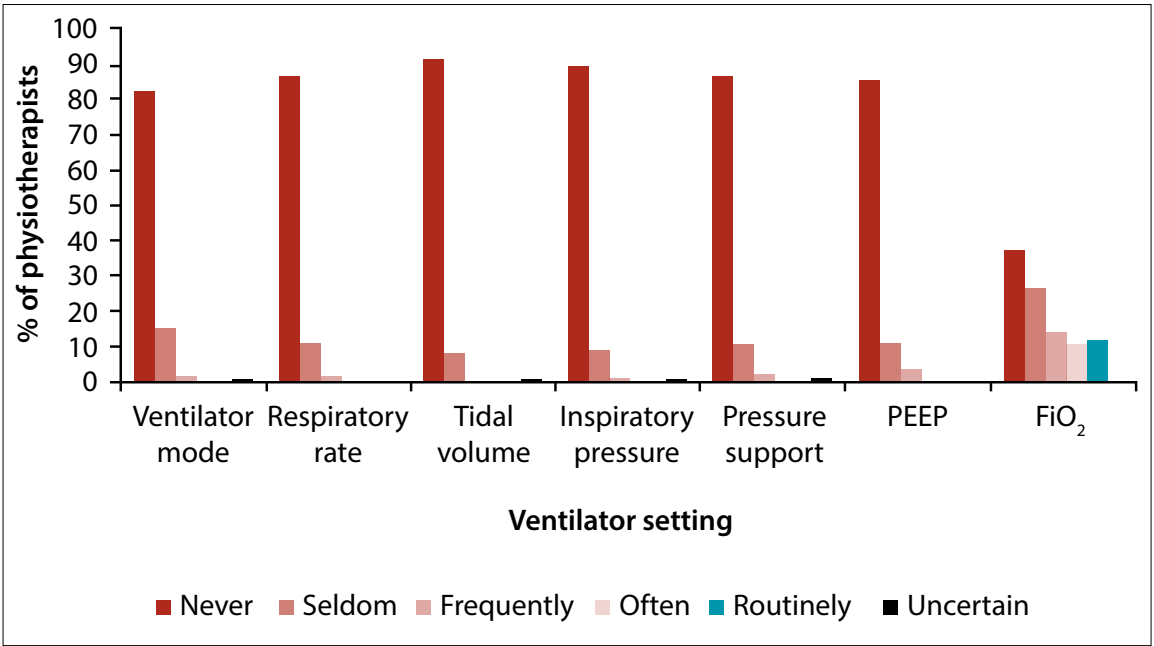

Fig. 1. Frequency with which respondents adjust MV settings.

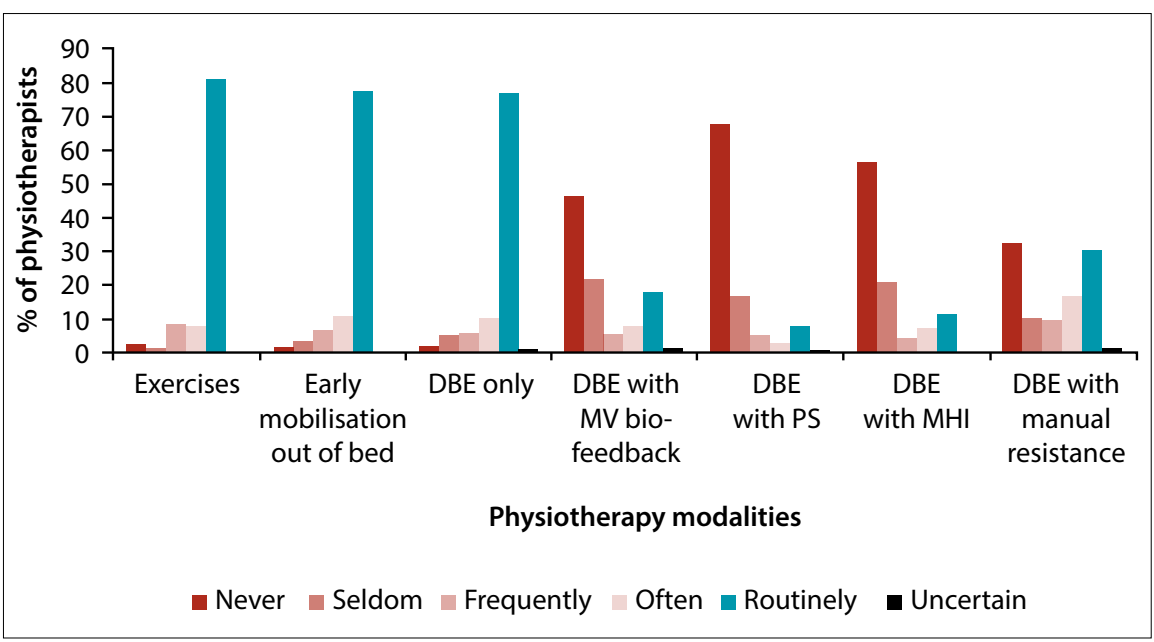

Fig. 2. Physiotherapy interventions used to facilitate respiratory muscle strengthening for patients on $M V$.

and their involvement in weaning $(p=0.43)$. Respondents qualified for $>11$ years were more routinely involved in the weaning of mechanically ventilated patients. There was no association between the type of physiotherapy degree respondents held and their involvement in weaning $(p=0.24)$.

Ninety-one per cent $(n=168)$ of respondents reported to be keen to receive further training in the role of physiotherapy in the management of mechanically ventilated patients to prepare them for weaning and extubation.

\section{Discussion}

The main findings of this survey were that some respondents were involved in the development of weaning protocols for the units that they work in, in decision-making about the initiation of weaning and in extubation, but the majority were not. Most respondents did not adjust MV settings, other than $\mathrm{FiO}_{2}$, during the process of weaning patients from $\mathrm{MV}$, but mostly used DBE, exercise therapy and early out-of-bed mobilisation to assist with weaning.

The majority of respondents held a Bachelor of Science degree in physiotherapy, worked in mixed ICU settings and in public sector hospitals, and had between 1 and 5 years of work experience in ICUs. All eight universities that offer physiotherapy qualifications in SA provide compulsory training to undergraduate physiotherapy students in the management of critically ill patients. The content of physiotherapy curricula pertaining to theoretical and clinical aspects of care of critically ill patients is, however, not standardised across universities owing to poorly defined minimum criteria for training set out by the Health Professions Council of SA (HPCSA); therefore, the content taught is often influenced by the amount of experience that academic staff have in the critical care setting. In 2012, the HPCSA put together a task team to develop criteria related to minimum standards of training for undergraduate 
physiotherapy students, the results of which are awaited. In addition, SA physiotherapy researchers with a special interest in critical care are in the process of developing minimum standards for physiotherapy practice in ICUs in an attempt to address this inequality in training. ${ }^{[12]}$ The low level of involvement of respondents in the development of weaning guidelines for their units and in decision-making about initiation of patient weaning from MV may, therefore, be ascribed to the differing levels of training received at undergraduate level about this aspect of patient care. Historically, SA physiotherapists have had a low level of involvement in the process of weaning patients from $\mathrm{MV}$, as reported by Van Aswegen and Potterton, ${ }^{[10]}$ who showed that only $12-15 \%$ of their respondents were involved in weaning of patients from MV in ICUs. This is in contrast to a European physiotherapy survey, which reported that $22 \%$ of respondents supervised weaning of patients from MV and 25\% performed extubation. ${ }^{[13]}$ Therefore, other members of the interprofessional team in an ICU might not deem it necessary for physiotherapists to be involved in the development of guidelines or in decision-making related to patient weaning. It is known that within the public healthcare sector in SA, new graduates fill most available posts and they do not work exclusively in the ICU. ${ }^{[12]}$ Even though a large number of respondents held senior positions, their relative inexperience in the ICU setting may account for their limited involvement in weaning of patients from MV.

Research published on the effects of non-physician-driven weaning and extubation protocols in the liberation of patients from MV stems mostly from the USA, involving respiratory therapists and nurses. ${ }^{[14]}$ Fewer studies have been published on the effects of physiotherapist involvement in the use of weaning and extubation protocols in $\mathrm{ICU}^{[7,11]}$ despite the fact that physiotherapists, and not respiratory therapists, work in critical care settings in various other countries. ${ }^{[8]}$ The prevalence of reporting on respiratory therapist involvement in weaning and extubation might, therefore, influence physicians' perceptions regarding the role that physiotherapists potentially play in this aspect of patient care. This perception is reflected by the low level of autonomy and decision-making in weaning patients from MV reported by respondents in this survey. In a large single-centred, randomised controlled trial from Italy, patients who received a nurse- and physiotherapist-lead weaning and extubation protocol had a significantly lower reintubation rate after extubation than those managed solely according to the physician's judgement. ${ }^{[7]}$ The authors stated that despite ICU staff reports that the protocol increased their work load, the nurses and physiotherapists reported their involvement in this protocol had a positive effect on their professional roles in the ICU. ${ }^{[7]}$ Plani et al. ${ }^{[11]}$ as mentioned previously, showed that physiotherapist and nurse involvement in a weaning and extubation protocol for patients with traumatic injury led to a clinically significant reduction in MV duration. The results of both of these studies suggest that physiotherapists who work in the ICU may have a valuable contribution to make towards weaning and extubation of patients from MV, especially in open-type ICU settings.

Respondents who had $>11$ years of ICU work experience were more likely to be involved in weaning patients from MV, according to the current survey. This suggests that with clinical experience, physiotherapists enhance their confidence and clinical reasoning skills and are therefore more likely to contribute meaningfully towards weaning practices in their units. Although few respondents in this survey adjusted MV settings for the purpose of respiratory muscle training during their daily practice, the majority indicated that they would be interested in receiving further education and training in the process of weaning of patients from MV. Such training might assist in raising the profile of physiotherapists in the provision of patient care in SA ICUs, especially in the public healthcare sector.

Exercise therapy, early out-of-bed mobilisation and DBE were routinely performed by the majority of respondents. It is known that muscle activity has an anti-inflammatory function ${ }^{[15]}$ and plays a beneficial role in the recovery of patients from critical illness by positively altering inflammatory cytokine activity ${ }^{[16]}$ Early mobilisation in intensive care and maintenance of muscle strength have been shown to reduce the number of ventilator-dependent days, shorten ICU and hospital length of stay and increase functional outcomes at hospital discharge.$^{[8,9]}$ Certain muscles of the upper limb insert onto and stabilise the rib cage. As these muscles are trained as part of whole-body exercise therapy, recruitment of accessory inspiratory muscles occurs, resulting in increased inspiratory muscle strength and more efficient ventilation patterns. ${ }^{[5]}$ The routine use of exercise therapy and early mobilisation by respondents in this survey, which affects global weakness associated with critical illness, to facilitate patient weaning from MV is therefore in keeping with evidence-based practice. . $^{[5,8,9]}$

Only some respondents in this survey performed extubation of patients. This finding is in contrast to that reported by Van Aswegen and Potterton, ${ }^{[10]}$ where $65 \%$ of physiotherapists in public and private sector hospitals indicated that they performed extubation. Possible explanations for this decreased involvement of physiotherapists in patient extubation could be the differing levels of training received at undergraduate level, relatively low levels of work experience in the ICU or because extubation is not the sole scope of practice of physiotherapists in SA, as nurses and doctors are also trained to perform this procedure. As physiotherapists in the public sector do not work exclusively in ICUs, ${ }^{[12]}$ other professionals may step in to perform extubation if the physiotherapist had left the unit prior to the orders for extubation being relayed.

A meta-analysis on the use of NIV for weaning showed that NIV use in recently extubated patients was associated with reduced ICU and hospital length of stay, incidence of pneumonia and reintubation rates. ${ }^{[17]}$ NIV was implemented after extubation by few respondents in this survey. Moran et al. ${ }^{[6]}$ investigated physiotherapy involvement in NIV hospital services in the British Isles using a survey study design. The authors reported that physiotherapists' involvement was mainly in the treatment of patients on NIV and not assessing a patient's need for or setting them up on NIV. They felt that these aspects of care were the responsibility of any appropriately trained member of the interprofessional team in the ICU and not solely that of the physiotherapist. ${ }^{[6]}$ This suggests that SA physiotherapists' limited involvement in implementation of NIV is in keeping with international practice.

\section{Study limitations}

The quality of research findings from survey-based studies is influenced by response rate. ${ }^{[18]}$ The study has a $57 \%$ non-response bias and therefore the results should be interpreted with caution. Findings were limited by the low response rate from physiotherapists working in private sector ICUs, the reasons for which are unknown.

The survey did not include physiotherapists' use of inspiratory muscle trainer devices to facilitate respiratory muscle training for weaning purposes. Research evidence suggests that inspiratory muscle training increases maximal inspiratory pressure and reduces MV and weaning time; ${ }^{[2]}$ therefore, future surveys should include this aspect of respiratory muscle training in the ICU. 


\section{Conclusion}

SA physiotherapists contribute towards weaning of patients from MV through prescription of exercise therapy, early out-of-bed mobilisation and DBE. Their involvement in adjustment of MV settings, decisionmaking regarding initiation of patient weaning and contributions towards development of weaning protocols for their units and extubation is limited. There is a need for further training of physiotherapists in the criteria for patient weaning and the additional methods employed to facilitate respiratory muscle training to ensure successful extubation.

\section{References}

. El-Khatib MF, Bou-Khalil P. Clinical review: Liberation from mechanical ventilation. Crit Care 2008;12(4):221. DOI:10.1186/cc6959

2. Gosselink R, Clerckx B, Robbeets C, et al. Physiotherapy in the intensive care unit. Neth J Crit Care 2011;15(2):66-75

3. Dantas CM, Dos Santos Silva PF, De Siqueira FHT, et al. Influence of early mobilisation on respiratory and peripheral muscle strength in critically ill patients. Rev Bras Ter Intensiva 2012;24(2):173-178. DOI:10.1590/S0103-507X2012000200013

4. Blackwood B, Alderdice F, Burns K, et al. Use of weaning protocols for reducing duration of mechanical ventilation in critically ill adult patients: Systematic review and meta-analysis. Br Med J 2011;10:342-356. DOI:10.1136/bmj.c7237

5. Martin UJ, Hincapie L, Nimchuk M, et al. Impact of whole-body rehabilitation in patients receiving chronic mechanical ventilation. Crit Care Med 2005;33(10):2259-2265. DOI:10.1097/01. $\mathrm{ccm} .0000181730 .02238 .9 \mathrm{~b}$

6. Moran FM, Bradley JM, Elborn JS, et al. Physiotherapy involvement in non-invasive ventilation hospital services: A British Isles survey. Int J Clin Pract 2005;59(4):453-456. DOI:10.1111/j.13685031.2005.00400.x
7. Navalesi P, Frigerio P, Moretti MP, et al. Rate of reintubation in mechanically ventilated neurosurgical and neurological patients: Evaluation of a systematic approach to weaning and extubation. Crit Care Med 2008;36(11):2986-2992. DOI:10.1097/CCM.0b013e31818b35f2

8. Ambrosino N, Makhabah DN. Comprehensive physiotherapy management in ARDS. Minerva Anestesiol 2013;79(5):554-563

9. Cameron S, Ball I, Cepinskas G, et al. Early mobilisation in the critical care unit: A review of adult and pediatric literature. J Crit Care 2015;30(4):664-672. DOI:10.1016/j. jcrc.2015.03.032

10. Van Aswegen H, Potterton J. A pilot survey of the current scope of practice of South African physiotherapists in intensive care units. S Afr J Physiother 2005;61:17-21

11. Plani N, Becker P, Van Aswegen $\mathrm{H}$. The use of a weaning and extubation protocol to facilitate effective weaning and extubation from mechanical ventilation in patients suffering from traumatic injuries: A non-randomized experimental trial comparing a prospective to retrospective cohort. Physiother Theory Pract 2013:29(3):211-221. DOI:10.3109/09593985.20 12.718410

12. Hanekom S, Van Aswegen H, Plani N, et al. Developing minimum clinical standards for physiotherapy in South African intensive care units: The nominal group technique in action. J Eval Clin Pract 2015;21(1):118-127. DOI:10.1111/jep.12257

13. Norrenberg M, Vincent JL. Brief report: A profile of European intensive care unit physiotherapists. Intensive Care Med 2000;26(7):988-994. DOI:10.1007/s001340051292

14. Haas CF, Loik PS. Ventilator discontinuation protocols. Respir Care 2012;57(10):1649-1662. DOI:10.4187/respcare.01895

15. Gleeson M, Bishop NC, Stensel DJ, et al. The anti-inflammatory effects of exercise: mechanisms and implications for the prevention and treatment of disease. Nat Rev Immunol 2011;11(9):607 615. DOI:10.1038/nri3041

16. Winkelman C, Johnson KD, Hejal R, et al. Examining the positive effects of exercise in intubated adults in ICU: A prospective repeated measures clinical study. Intensive Crit Care Nurs 2012;28(6):307-320. DOI:10.1016/j.iccn.2012.02.007

17. Glossop AJ, Shepherd N, Bryden DC, et al. Non-invasive ventilation for weaning, avoiding reintubation after extubation and in the postoperative period: A meta-analysis. $\mathrm{Br} \mathrm{J}$ Anaesth 2012;109(3):305-314. DOI:10.1093/bja/aes270

18. Fincham JE. Response rates and responsiveness for surveys, standards and the journal. Am J Pharm Educ 2008;72(2):43. DOI:10.5688/aj72024 